\title{
Temporal and Spatial Variation of Synchrotron X-ray Stripes in Tycho's Supernova Remnant
}

\author{
Masamune Matsuda ${ }^{1}$, Takaaki TanAKA ${ }^{1}$, Hiroyuki UChIdA ${ }^{1}$, Yuki Amano ${ }^{1}$ \\ and Takeshi Go Tsuru ${ }^{1}$
}

\author{
${ }^{1}$ Department of Physics, Kyoto University, Kitashirakawa Oiwake-cho, Sakyo, Kyoto, Kyoto \\ 606-8502, Japan
}

*E-mail: matsuda.masamune.38a@kyoto-u.jp

Received 2020 April 4; Accepted 2020 July 11

\begin{abstract}
The synchrotron X-ray "stripes" discovered in Tycho's supernova remnant (SNR) have been attracting attention since they may be evidence for proton acceleration up to $\mathrm{PeV}$. We analyzed Chandra data taken in 2003, 2007, 2009, and 2015 for imaging and spectroscopy of the stripes in the southwestern region of the SNR. Comparing images obtained at different epochs, we find that time variability of synchrotron X-rays is not limited to two structures previously reported but is more common in the region. Spectral analysis of nine bright stripes reveals not only their time variabilities but also a strong anti-correlation between the surface brightness and photon indices. The spectra of the nine stripes have photon indices of $\Gamma=2.1-2.6$ and are significantly harder than those of the outer rim of the SNR in the same region with $\Gamma=2.7-2.9$. Based on these findings, we indicate that the magnetic field is substantially amplified, and suggest that particle acceleration through a stochastic process may be at work in the stripes.
\end{abstract}

Key words: acceleration of particles — ISM: individual objects (SN1572, Tycho's SNR) — ISM: supernova remnant - magnetic fields $-X$-rays: ISM

\section{Introduction}

Supernova remnants (SNRs) have long been hypothesized to be major production sites of Galactic cosmic rays up to the so-called knee in the cosmic-ray spectrum at $\sim 3 \mathrm{PeV}$. The hypothesis is favored partly because diffusive shock acceleration, expected be working at the expanding shock of SNRs, can naturally explain the power-law spectrum of cosmic rays (e.g., Blandford \& Eichler 1987; Malkov \& Drury 2001). Energetics is another major reason why SNRs have been regarded as cosmic-ray origin. If $\sim 10 \%$ of kinetic energy released in supernova explosions is supplied to particle acceleration, SNRs can support the energy density of cosmic rays in the interstellar space.

Detecting synchrotron radiation, X-ray observations have been providing evidence that electrons are accelerated to ultra-relativistic energies at blast waves of young SNRs (e.g., Koyama et al. 1995; Tanaka et al. 2008). Gamma-ray emis- sions, although their radiation mechanisms sometimes are subject to considerable debate, serve as direct evidence for particle acceleration in SNRs (e.g., Aharonian et al. 2007). Recent sensitive GeV gamma-ray observations with the Fermi Large Area Telescope and AGILE enabled identification of $\pi^{0}$-decay emission in SNRs, offering a compelling way of probing acceleration of protons and nuclei, the primary component of Galactic cosmic rays (Ackermann et al. 2013; Jogler \& Funk 2016; Giuliani et al. 2011). In spite of the mounting evidence, however, clear indication of acceleration up to the knee has not been obtained from SNRs so far (e.g., Archambault et al. 2017).

Protons should be accelerated up to the knee if SNRs are indeed the origins of Galactic cosmic rays. Lagage \& Cesarsky (1983) estimated the maximum energy of particles in SNR shocks as $E_{\max }=100 Z(B / 1 \mu \mathrm{G}) \mathrm{TeV}$, where $Z$ and $B$ are the particle charge and magnetic field strength, respectively. The equation implies that the magnetic field should be sub- 
stantially amplified in order for protons gain energies up to the knee. Theoretical studies indeed indicate that cosmic-ray streaming instability can lead to significant amplification of magnetic field at a strong shock (e.g., Bell \& Lucek 2001; Bell 2004). Observationally, thin filamentary structures (e.g., Bamba et al. 2003; Warren et al. 2005) as well as rapid variability of synchrotron radiation (Uchiyama et al. 2007; Uchiyama \& Aharonian 2008; Borkowski et al. 2018; Okuno et al. 2020) discovered in X-ray data of SNRs are often interpreted as the results of magnetic field amplification.

The synchrotron X-ray “stripes” in Tycho's SNR discovered by Eriksen et al. (2011) may be indicative of presence of PeV particles as well as magnetic field amplification. Eriksen et al. (2011) interpreted that the gaps between the stripes $\left(8^{\prime \prime}\right)$ corresponds to twice the gyroradius of accelerated protons, concluding that protons reach PeV energies. Okuno et al. (2020) discovered time variable features in the southwestern region of the SNR where stripes are observed. The time variability can be explained if the magnetic field is amplified to $\sim 100 \mu \mathrm{G}$ and/or if magnetic turbulence significantly changes with time. The discovery of the stripes triggered some theoretical works proposing models for the peculiar structure (e.g., Bykov et al. 2011; Malkov et al. 2012; Caprioli \& Spitkovsky 2013; Laming 2015). Yet, their physical origin still is an open question.

Here we report imaging and spectral studies of the southwestern stripes of Tycho's SNR using Chandra archival data. We aim to reveal temporal and spatial variation of synchrotron $\mathrm{X}$-rays of the stripes to discuss their physical origin. While Okuno et al. (2020) focused on two specific features, we characterize spectral and temporal behavior of each stripe in a more systematical way. Throughout the paper, the statistical errors are quoted at the $1 \sigma$ level.

\section{Observations and Data Reduction}

We analyzed Chandra ACIS data of Tycho's SNR obtained in 2003, 2007, 2009, and 2015. Table 1 presents the observation log. We reprocessed and screened all the data with chandra_repro in CIAO version 4.11 with CALDB version 4.8.2. The effective exposures after the screening are shown in table 1 . We performed relative astrometric corrections to each dataset. We first detected point sources in the field with wavdetect in CIAO. We then reprojected each events file by cross matching the detected sources with wcs_match and wcs_update tasks of CIAO. Low photon statistics did not allow us to detect enough sources to perform the astrometric corrections for observations ObsID = 8551, 0903, 10904, and 10906 . We therefore discarded these datasets for the imaging analysis, where accurate corrections are required. For the spectral analysis, we used these datasets for better statistics. We co-added datasets obtained in the same year. The total effective times for
Table 1. Observation log.

\begin{tabular}{ccc}
\hline ObsID & Start date & $\begin{array}{c}\text { Effective exposure } \\
(\mathrm{ks})\end{array}$ \\
\hline 3837 & $2003-04-29$ & 146 \\
7639 & $2007-04-23$ & 109 \\
8551 & $2007-04-26$ & 33 \\
10093 & $2009-04-13$ & 118 \\
10094 & $2009-04-18$ & 90 \\
10095 & $2009-04-23$ & 173 \\
10096 & $2009-04-27$ & 106 \\
10097 & $2009-04-11$ & 107 \\
10902 & $2009-04-15$ & 40 \\
10903 & $2009-04-17$ & 24 \\
10904 & $2009-04-13$ & 35 \\
10906 & $2009-05-03$ & 41 \\
15998 & $2015-04-22$ & 147 \\
\hline
\end{tabular}

the imaging analysis in 2003, 2007, 2009, and 2015 are $146 \mathrm{ks,}$ $109 \mathrm{ks}, 634 \mathrm{ks}$, and $147 \mathrm{ks}$, respectively, while those for the spectral analysis are 146 ks, 142 ks, 734 ks, and 147 ks.

\section{Analysis and Results}

\subsection{Imaging Analysis}

Figure 1a shows a difference image of Tycho's SNR constructed by subtracting an exposure-corrected image taken in 2003 from that in 2015. A zoom-in view of the western region is given in figure $1 \mathrm{~b}$. We also present exposure-corrected images from each year in figure 1d. We selected an energy band of 4-6 keV, where synchrotron emission is dominant (e.g., Eriksen et al. 2011). Most of the stripe features appear to move outward as the outer rim, which most probably corresponds to the blast waves (Warren et al. 2005), expands. However, some of the changes cannot simply be accounted for just by the expansion. The stripes along the green curves in figure 1c generally become brighter in 2015. We note that most of the brightening stripes are too faint to be seen in the exposure-corrected images in figure $1 \mathrm{~d}$. On the other hand, the emission along the cyan line in figure 1c shows a flux decrease. These results indicate that the small knot structure, which Okuno et al. (2020) reported, is not the only structure that shows brightening over the years in this region. We also found hints of the proper motions of the faint stripes along the direction perpendicular to the shock normal.

Figure $2 \mathrm{~b}$ shows projections along the azimuthal direction from the rectangular region in figure $2 \mathrm{a}$, which includes the brightest stripe (S7 in figure 1c). The sharp and broad peaks correspond to the rim and the stripe, respectively. Both peaks clearly are moving outward with time as already suggested by the difference image in figure 1a. We first measured the proper motion of the rim. Artificially shifting the profile in 2003, we calculated $\chi^{2}$ between the shifted profile in 2003 and the observed profile in 2015 in the range of $66^{\prime \prime}-90^{\prime \prime}$, and searched for 
(a)

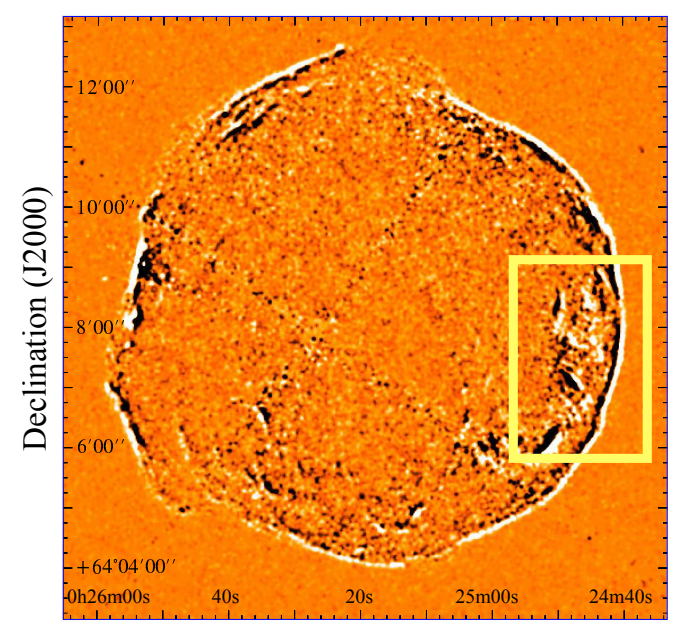

Right ascension (J2000) (b)

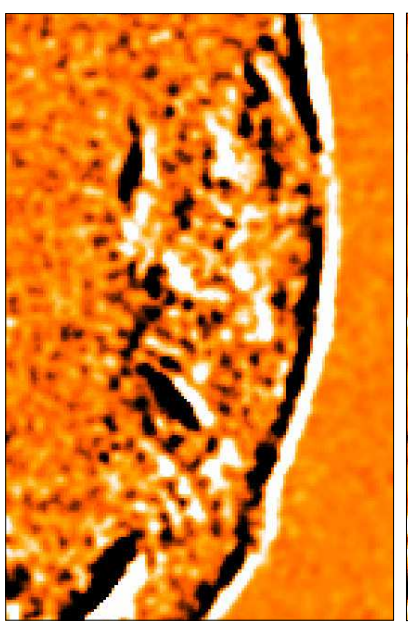

(c)

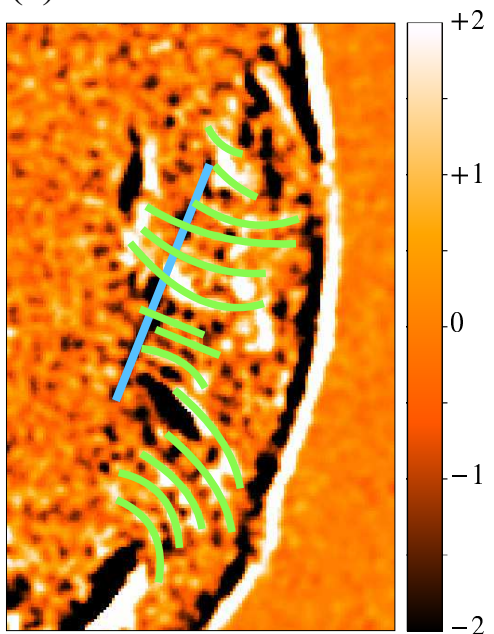

(d)
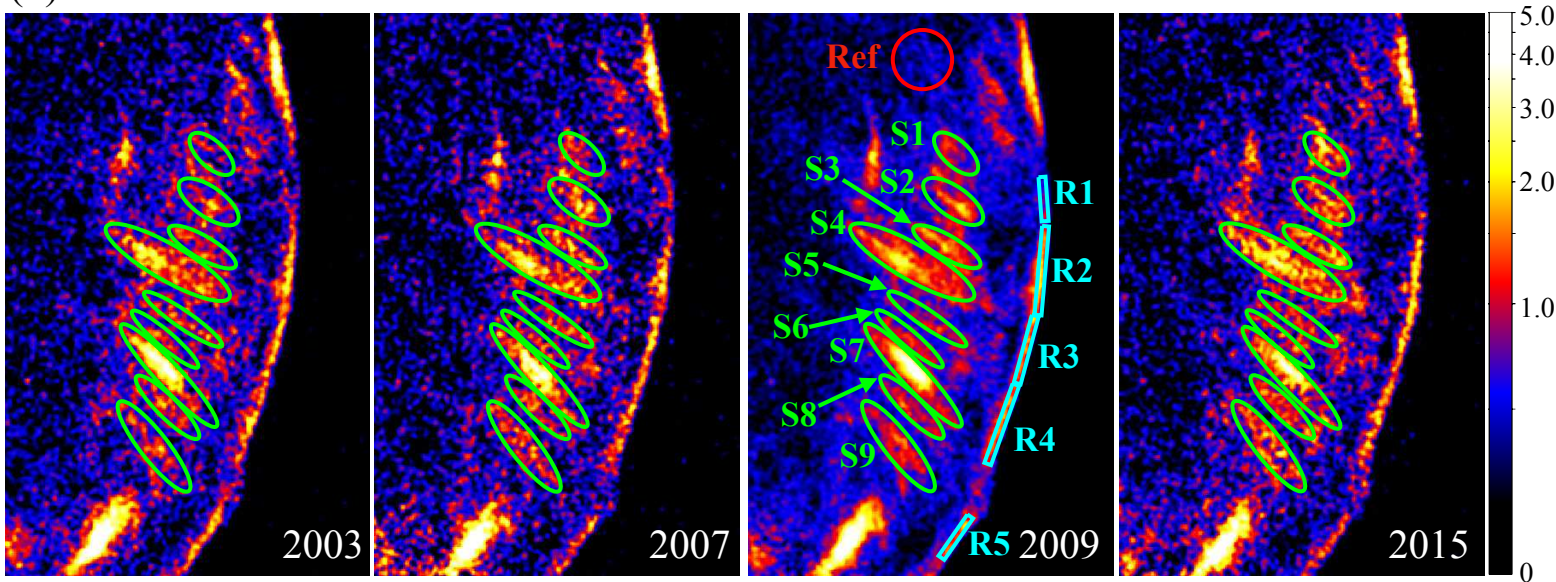

Fig. 1. (a) Difference image between 2003 and 2015 in the 4-6 keV band. The green box corresponds to the region shown in panels (b), (c), and (d). (b) Zoom-in view of the yellow box in panel (a). (c) Same as panel (b) but with guides for identification of notable features overlaid. (d) Exposure-corrected Chandra ACIS images of Tycho's SNR in 2003, 2007, 2009, and 2015. The energy band is 4-6 keV. The regions S1-S9 and R1-R5 are those for the spectral analysis. The Ref region is used for estimating the parameters for the thermal component of the emissions of the stripes. In all the panels, the unit for the color scale is $10^{-8} \mathrm{ph} \mathrm{s}^{-1} \mathrm{~cm}^{-2}$.

a shift that gives the minimum $\chi^{2}$. As a result, we obtained a velocity of $0.29 \pm 0.01$ arcsec $\mathrm{yr}^{-1}$, which can be translated into $3400 \pm 100 \mathrm{~km} \mathrm{~s}^{-1}$ with a distance of $2.5 \mathrm{kpc}$ assumed (Zhou et al. 2016). We note that Williams et al. (2016) reported a proper motion consistent with ours (The region we analyzed roughly coincides with their Reg 13). Measurement of the proper motion of the stripe, on the other hand, was found to be difficult because of its time-variable shape. A closer look at the profiles plotted in figure $2 \mathrm{~b}$ suggests that the peak corresponding to the stripe becomes narrower in 2015 than in 2003. This is more clearly visible in smoothed profiles presented in figure 3 . In this figure, we shifted the profile in 2003, assuming a velocity of

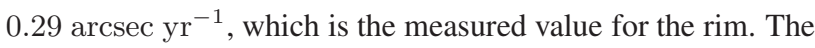
locations of the peaks are roughly consistent with each other, leading to a conclusion that the proper motion of the stripe is at the same level as the rim.

\subsection{Spectral Analysis}

We performed spectroscopy of each bright stripe by extracting spectra from the nine regions labeled as S1-S9 in figure 1c. Backgrounds are extracted from the outside of the SNR within the ACIS-I array. The spectra were binned so that each bin has at least 10 counts and were fitted over the $0.5-10 \mathrm{keV}$ energy band with XSPEC 12.10.1f (Arnaud 1996). Following the works by Sato, \& Hughes (2017), Yamaguchi et al. (2017), and Okuno et al. (2020), we fitted the spectra with a model consisting of non-thermal and thermal components. We modeled the non-thermal component with a power law. To the thermal emission, which can be attributed to the supernova ejecta, we ap- 
Table 2. Best-fit parameters in 2009

\begin{tabular}{|c|c|c|c|c|c|c|c|c|c|}
\hline Parameters (unit) & $\mathrm{S} 1$ & $\mathrm{~S} 2$ & S3 & $\mathrm{S} 4$ & S5 & S6 & S7 & S8 & S9 \\
\hline Solid angle $\left(\operatorname{arcsec}^{2}\right)$ & 143.8 & 210.2 & 172.7 & 505.3 & 161.7 & 141.1 & 338.8 & 183.4 & 311.6 \\
\hline$N_{\mathrm{H}}\left(10^{21} \mathrm{~cm}^{-2}\right)$ & $6.80 \pm 0.17$ & $6.37_{-0.10}^{+0.26}$ & $5.23_{-0.12}^{+0.21}$ & $5.83_{-0.06}^{+0.05}$ & $5.92_{-0.20}^{+0.24}$ & $6.32_{-0.19}^{+0.30}$ & $6.03_{-0.12}^{+0.17}$ & $6.56_{-0.28}^{+0.20}$ & $5.26_{-0.12}^{+0.14}$ \\
\hline \multicolumn{10}{|l|}{ Power law } \\
\hline $\bar{\Gamma}$ & $2.39 \pm 0.04$ & $2.37_{-0.03}^{+0.04}$ & $2.06 \pm 0.03$ & $2.15 \pm 0.01$ & $2.44 \pm 0.03$ & $2.40 \pm 0.04$ & $2.12_{-0.03}^{+0.02}$ & $2.25_{-0.03}^{+0.02}$ & $2.55 \pm 0.02$ \\
\hline Flux* & $0.43 \pm 0.01$ & $0.77 \pm 0.01$ & $0.76 \pm 0.01$ & $2.35 \pm 0.02$ & $0.50 \pm 0.01$ & $0.46 \pm 0.01$ & $2.08_{-0.01}^{+0.02}$ & $0.64 \pm 0.01$ & $1.05_{-0.02}^{+0.01}$ \\
\hline \multicolumn{10}{|l|}{ IME compconent } \\
\hline $\begin{array}{l}\text { Norm. } .^{\dagger} \\
\left(10^{9} \mathrm{~cm}^{-5}\right)\end{array}$ & $3.31_{-0.38}^{+0.42}$ & $1.48_{-0.43}^{+0.41}$ & $1.71_{-0.36}^{+0.24}$ & $4.97_{-0.26}^{+0.86}$ & $2.11_{-0.40}^{+0.70}$ & $1.51_{-0.38}^{+0.54}$ & $1.50_{-0.61}^{+0.49}$ & $2.22_{-0.42}^{+0.50}$ & $0.35_{-0.04}^{+0.06}$ \\
\hline$k T_{\mathrm{e}}(\mathrm{keV})$ & $1.49_{-0.03}^{+0.04}$ & $1.65_{-0.05}^{+0.03}$ & $1.68_{-0.04}^{+0.01}$ & $1.55 \pm 0.01$ & $1.58_{-0.02}^{+0.03}$ & $1.57_{-0.03}^{+0.04}$ & $1.71_{-0.04}^{+0.03}$ & $1.45_{-0.04}^{+0.01}$ & $1.41 \pm 0.03$ \\
\hline $\begin{array}{l}n_{\mathrm{e}} t \\
\left(10^{10} \mathrm{~s} \mathrm{~cm}^{-3}\right)\end{array}$ & \multicolumn{9}{|c|}{4.53 (fixed) } \\
\hline$[\mathrm{Mg} / \mathrm{C}] /[\mathrm{Mg} / \mathrm{C}]_{\odot}$ & $4.8_{-0.4}^{+0.6}$ & $7.2 \pm 1.4$ & $9.2_{-0.8}^{+1.9}$ & $8.1_{-0.7}^{+0.4}$ & $5.2_{-1.3}^{+0.3}$ & $6.4_{-1.6}^{+1.7}$ & $10.6_{-2.1}^{+6.9}$ & $5.5_{-0.9}^{+0.8}$ & $4.8 \pm 0.6$ \\
\hline$[\mathrm{Si} / \mathrm{C}] /[\mathrm{Si} / \mathrm{C}]_{\odot}$ & $84_{-9}^{+10}$ & $161_{-35}^{+68}$ & $190_{-27}^{+40}$ & $238_{-22}^{+23}$ & $183_{-37}^{+4.3}$ & $189_{-46}^{+38}$ & $279_{-69}^{+194}$ & $112_{-20}^{+33}$ & $73_{-9}^{+10}$ \\
\hline$[\mathrm{S} / \mathrm{C}] /[\mathrm{S} / \mathrm{C}] \odot$ & $77_{-8}^{+10}$ & $130_{-28}^{+67}$ & $143_{-19}^{+31}$ & $185_{-20}^{+11}$ & $134_{-28}^{+30}$ & $139_{-36}^{+39}$ & $194_{-48}^{+144}$ & $91_{-16}^{+25}$ & $81 \pm 11$ \\
\hline$[\mathrm{Ar} / \mathrm{C}] /[\mathrm{Ar} / \mathrm{C}] \odot$ & $85_{-11}^{+14}$ & $105_{-12}^{+40}$ & $110_{-10}^{+13}$ & $175_{-11}^{+8}$ & $99_{-9}^{+24}$ & $106_{-22}^{+31}$ & $202_{-65}^{+140}$ & $77 \pm 9$ & $86_{-13}^{+14}$ \\
\hline$[\mathrm{Ca} / \mathrm{C}] /[\mathrm{Ca} / \mathrm{C}] \odot$ & $156_{-25}^{+28}$ & $202_{-26}^{+72}$ & $158_{-25}^{+55}$ & $163_{-29}^{+20}$ & $159_{-23}^{+24}$ & $258_{-77}^{+86}$ & $298_{-77}^{+93}$ & $142 \pm 24$ & $168_{-30}^{+33}$ \\
\hline \multicolumn{10}{|l|}{ Fe component } \\
\hline $\begin{array}{l}\text { Norm. } .^{\dagger} \\
\left(10^{9} \mathrm{~cm}^{-5}\right)\end{array}$ & $3.31^{\dagger}$ & $1.48^{\dagger}$ & $1.77^{\dagger}$ & $4.97^{\dagger}$ & $2.11^{\dagger}$ & $1.51^{\dagger}$ & $1.50^{\dagger}$ & $2.22^{\dagger}$ & $0.37^{\dagger}$ \\
\hline$k T_{\mathrm{e}}(\mathrm{keV})$ & $5.54_{-0.83}^{+0.91}$ & $4.30_{-0.98}^{+1.30}$ & $3.85_{-0.80}^{+1.20}$ & $4.63_{-0.26}^{+0.40}$ & $2.36_{-0.39}^{+0.35}$ & $3.00_{-0.83}^{+1.84}$ & $2.04_{-0.26}^{+0.62}$ & $2.41_{-0.55}^{+0.26}$ & $1.53_{-0.23}^{+0.12}$ \\
\hline $\begin{array}{l}n_{\mathrm{e}} t \\
\left(10^{10} \mathrm{~s} \mathrm{~cm}^{-3}\right)\end{array}$ & \multicolumn{9}{|c|}{0.74 (fixed) } \\
\hline $\begin{array}{l}{[\mathrm{Fe} / \mathrm{C}] /[\mathrm{Fe} / \mathrm{C}]_{\odot}} \\
\left(=[\mathrm{Ni} / \mathrm{C}] /[\mathrm{Ni} / \mathrm{C}]_{\odot}\right)\end{array}$ & $3.6_{-0.4}^{+0.5}$ & $5.4_{-1.2}^{+1.9}$ & $5.4_{-0.5}^{+1.4}$ & $9.2_{-1.0}^{+0.8}$ & $7.6_{-1.1}^{+1.4}$ & $5.5 \pm 1.2$ & $8.0_{-1.5}^{+3.2}$ & $3.8_{-0.5}^{+0.8}$ & $4.8_{-0.5}^{+0.6}$ \\
\hline$\underline{\text { Gaussian }}$ & & & & & & & & & \\
\hline Norm..$^{\ddagger}$ & $0.53 \pm 0.05$ & $0.33 \pm 0.05$ & $0.36 \pm 0.04$ & $1.40_{-0.07}^{+0.08}$ & $0.60 \pm 0.05$ & $0.47 \pm 0.05$ & $0.44_{-0.07}^{+0.06}$ & $0.35_{-0.05}^{+0.04}$ & $0.61_{-0.07}^{+0.08}$ \\
\hline Centroid (keV) & $1.25_{-0.01}^{+0.02}$ & $1.28_{-0.02}^{+0.05}$ & $1.24 \pm 0.01$ & $1.253_{-0.001}^{+0.006}$ & $1.238_{-0.007}^{+0.002}$ & $1.234 \pm 0.003$ & $1.232_{-0.002}^{+0.022}$ & $1.254_{-0.005}^{+0.007}$ & 1.23 (fixed) \\
\hline$\chi^{2}($ d.o.f. $)$ & $405(291)$ & $375(331)$ & $463(338)$ & $1153(433)$ & $593(302)$ & $451(299)$ & $598(418)$ & $392(321)$ & $606(373)$ \\
\hline
\end{tabular}

plied a two-component non-equilibrium ionization (NEI) model using the vnei model in XSPEC. We employed the TuebingenBoulder absorption model (TBabs; Wilms et al. 2000) for interstellar absorption.

One of the two NEI components represents the emission from $\mathrm{Fe}$ whereas the other is for intermediate-mass elements (IMEs: $\mathrm{Mg}, \mathrm{Si}, \mathrm{S}, \mathrm{Ar}$, and $\mathrm{Ca}$ ). We treated the abundances of IMEs and $\mathrm{Fe}$ as free parameters, and linked the abundance of $\mathrm{Ni}$ to Fe. Since Tycho's SNR is of type Ia origin, the abundances of $\mathrm{H}, \mathrm{He}$, and $\mathrm{N}$ were fixed to zero. The abundances of $\mathrm{O}$ and $\mathrm{Ne}$ with respect to $\mathrm{C}$ were fixed at the solar values because $\mathrm{C}$ has the lowest atomic number in the elements that would be present in the ejecta. Emission measures of the Fe and IME components ( $\left.\equiv \frac{1}{[\mathrm{C} / \mathrm{H}]_{\odot}} \frac{1}{4 \pi d^{2}} \int n_{\mathrm{e}} n_{\mathrm{C}} d V\right)$ were linked to each other, where $d$ is the distance to Tycho's SNR, $n_{\mathrm{e}}$ and $n_{\mathrm{C}}$ are number densities of electron and carbon, and $V$ is the volume of the emitting plasma. Fitting the spectra, we found residuals at $\sim 1.2 \mathrm{keV}$, which are also seen in spectra of Tycho's SNR (e.g., Sato, \& Hughes 2017; Okuno et al. 2020) as well as other SNRs (e.g., Okon et al. 2019). Although the cause of the residuals is not clear (see a discussion by Okon et al. 2019), we added a Gaussian to the model to improve the fits. The centroid energy of the Gaussian was allowed to vary for spectra from all the regions except for $\mathrm{S} 9$. We fixed it to $1.23 \mathrm{keV}$ for $\mathrm{S} 9$ since it cannot be well constrained.

Strong non-thermal emission of the stripes makes it difficult to determine the parameters for the NEI components. To constrain the parameters, therefore, we analyzed a spectrum extracted from the region with less contribution from the nonthermal component, which is labeled as "Ref" in figure 1c. Since the region is located at a similar radius of the SNR to stripes, we assumed that ionization ages $\left(n_{e} t\right)$ of the NEI components are common between the "Ref" region and stripes, and determined them by fitting the "Ref" spectrum. The spectral fitting yielded $n_{\mathrm{e}} t=4.52 \times 10^{10} \mathrm{~s} \mathrm{~cm}^{-3}$ and $n_{\mathrm{e}} t=0.74 \times 10^{10}$ $\mathrm{s} \mathrm{cm}^{-3}$ for the IME and Fe components, respectively. We fixed $n_{\mathrm{e}} t$ to these values when fitting the spectra of the stripe regions.

We first fitted spectra from observations in 2009, which have the highest statistics thanks to the longest exposure time. The spectra are plotted with the best-fit models in figure 4, and the best-fit parameters are summarized in table 2. We then fitted spectra from 2003, 2007, and 2015 observations to see time variability of the stripe emissions. The parameters for the thermal components were fixed to those obtained for the spectra in 2009 except for the emission measures. In figure 5, we plot surface brightness of the non-thermal component as a function of photon index, which reveals a significant stripe-to-stripe variation of the parameters as well as time variability of each stripe. 
(a)
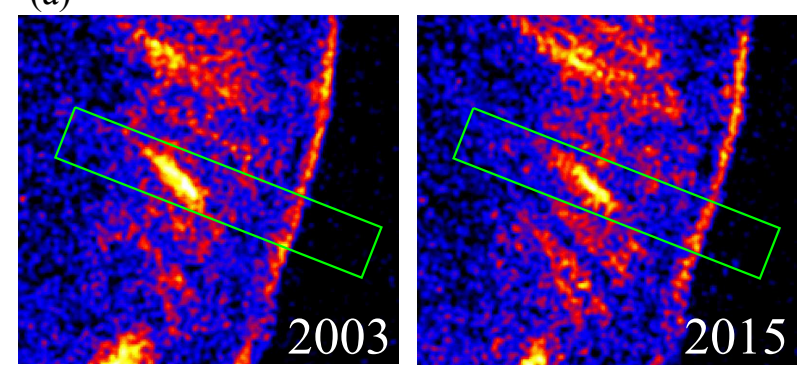

(b)

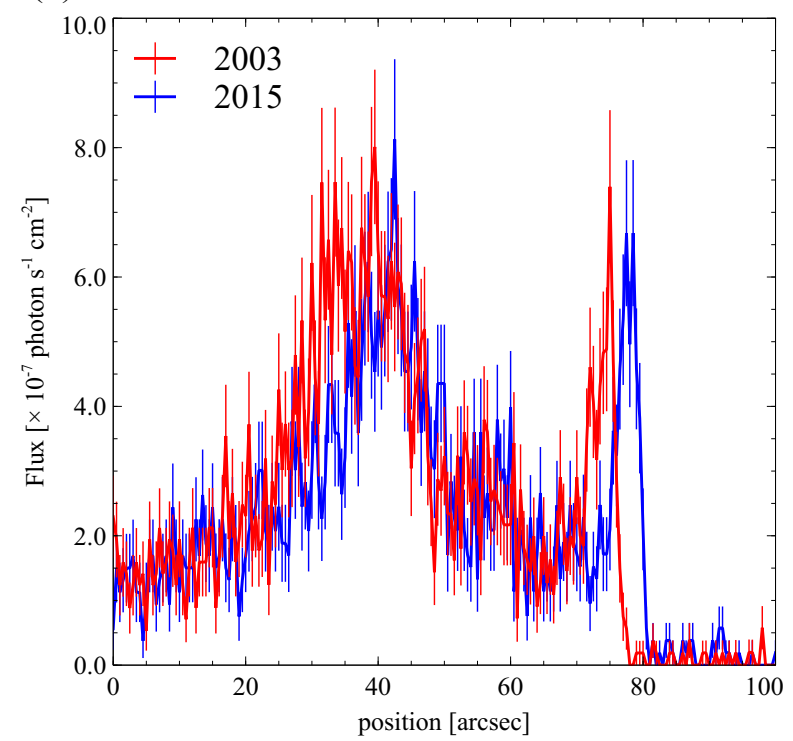

Fig. 2. (a) Regions used for the projection in panel (b). (b) Profiles extracted from the regions shown in panel (a). The widths of each bin are 0 !' $^{\prime}$. The geometrical center of the SNR is to the left.

Another finding to note here is the strong anti-correlation between surface brightness and photon indices of the stripe emission.

\section{Discussion}

Our imaging and spectral analyses have revealed time variable nature of the stripes in Tycho's SNR. Although Okuno et al. (2020) reported time variability only of two structures, including $\mathrm{S} 1$ in our definition, similar variability seems to be rather universal in this part of the SNR. Figure 5 indeed indicates significant flux variability of $\mathrm{S} 2, \mathrm{~S} 3, \mathrm{~S} 7$, and $\mathrm{S} 9$, in addition to $\mathrm{S} 1$. The fluxes of the stripes S2 and S3 are lower in 2003 than in the other years. The stripe S7 underwent a flux brightening from 2003 to 2007 and then decayed. In the case of S9, a continuous flux increase was observed from 2003 to 2015. The images in figure 1 furthermore indicate not only the bright stripes but also much fainter structures are also variable.

Following Uchiyama et al. (2007), Uchiyama \& Aharonian (2008), and Okuno et al. (2020), we can estimate the magnetic

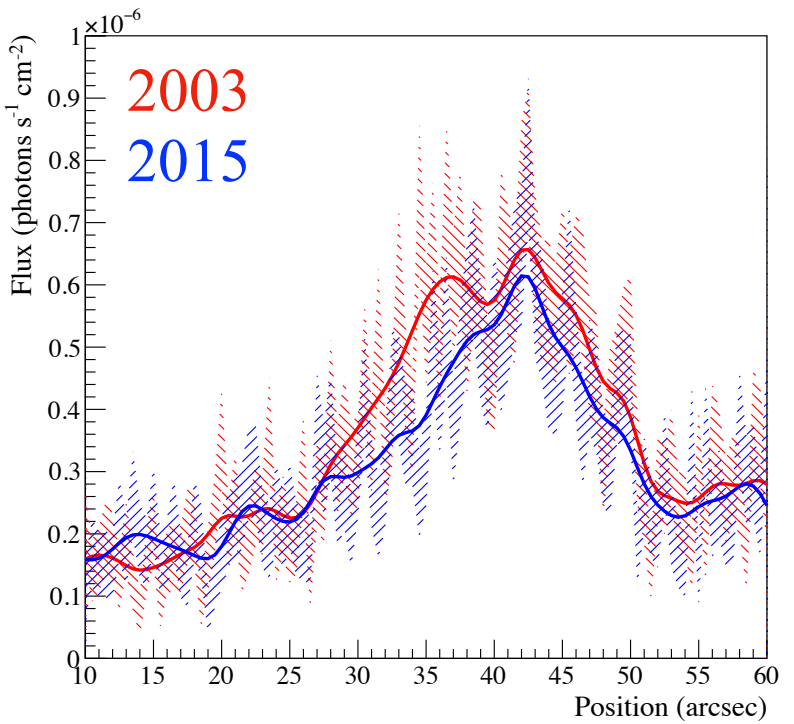

Fig. 3. Comparison between the profile in 2015 and the moved profile in 2003 by 3 .' $^{\prime}$. Oblique lines correspond to the range of $1 \sigma$. Solid lines show smoothed profiles with bandwidth $=3$.

field strength of the emitting region if we attribute the brightening to production of relativistic electrons through acceleration and the flux decay to decrease of electrons emitting synchrotron $\mathrm{X}$-rays. Assuming diffusive shock acceleration, we can write the acceleration timescale as

$t_{\mathrm{acc}}=4 \eta\left(\frac{\varepsilon}{\mathrm{keV}}\right)^{0.5}\left(\frac{B}{400 \mu \mathrm{G}}\right)^{-1.5}\left(\frac{v_{\mathrm{sh}}}{3400 \mathrm{~km} \mathrm{~s}^{-1}}\right)^{-2} \mathrm{yr}(1)$ where $\eta(\geq 1)$ is the so-called "gyrofactor", $\varepsilon$ is synchrotron photon energy, $B$ is the magnetic field strength, and $v_{\mathrm{sh}}$ is the shock velocity. We assumed $v_{\mathrm{sh}}=3400 \mathrm{~km} \mathrm{~s}^{-2}$ according to our proper motion measurement in $\S 3.1$. The synchrotron cooling timescale can be given as

$t_{\text {syn }}=4\left(\frac{\varepsilon}{\mathrm{keV}}\right)^{-0.5}\left(\frac{B}{500 \mu \mathrm{G}}\right)^{-1.5} \mathrm{yr}$.

Since we observed flux changes in a timescale of several years, the above equations lead to a conclusion that the magnetic field in the stripe region is $\sim 500 \mu \mathrm{G}$. We note here that this estimate would have some uncertainties. The above equations assume that electrons with a certain energy emit monochromatic synchrotron photons, which is not true in reality and hence produces uncertainties.

The 3D location of the stripes would be key information for discussing their physical origin. In the above magnetic field strength estimation, we implicitly assumed that the stripes are on the projected blast waves of the SNR. In this case, proper motion of the stripes should appear to be slower than the expansion velocity since only the transverse velocity component is observed. The transverse velocity is calculated to be 0.25 arcsec $\mathrm{yr}^{-1}$, assuming spherical shell expansion with a velocity of $0.29 \operatorname{arcsec}_{\mathrm{yr}^{-1}}$ (§3.1). We found the difference of 


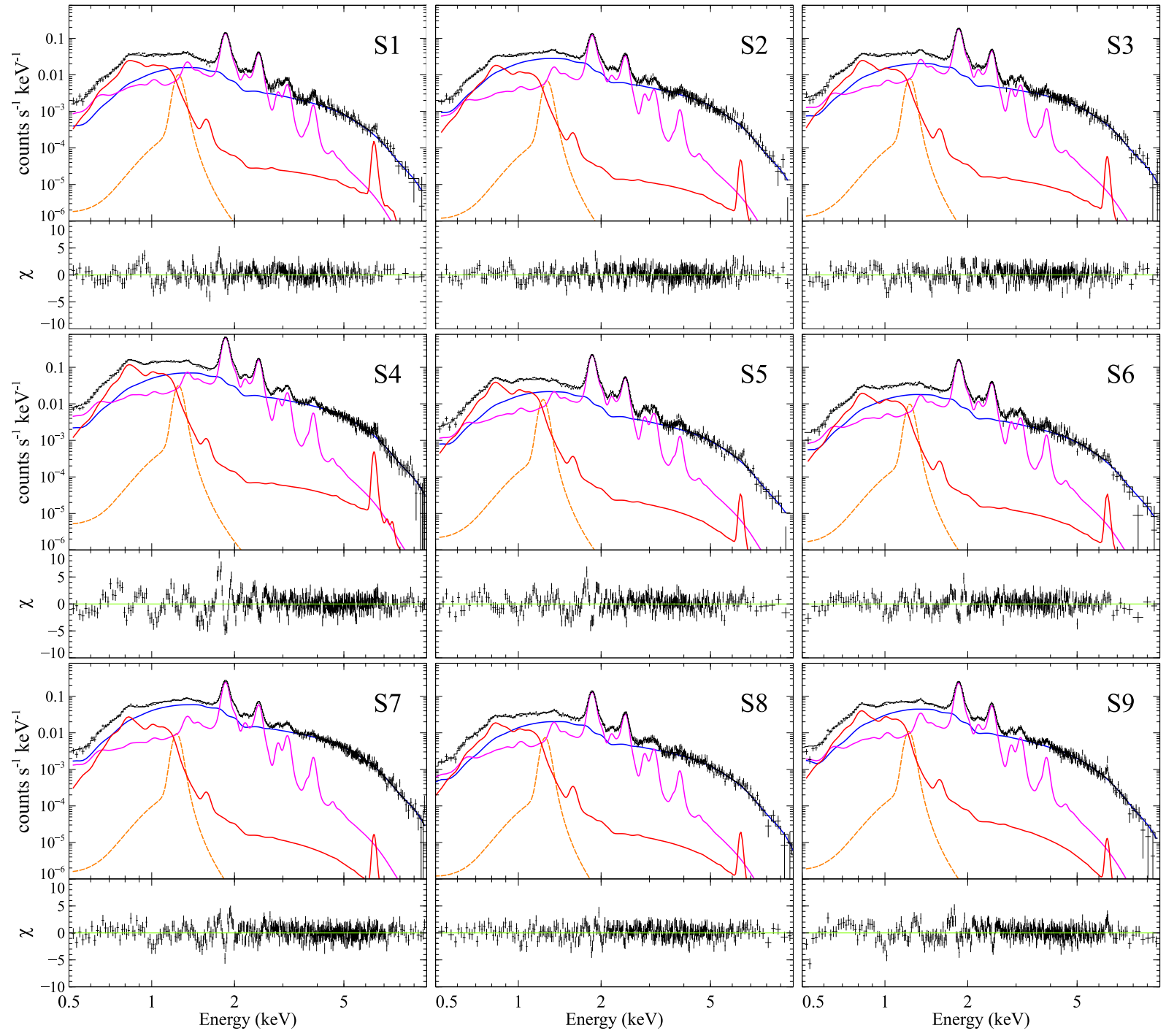

Fig. 4. Spectra and the best-fit models for regions S1-S9 obtained from the data taken in 2009. The black curves are the sums of all the components whereas the other colors show contributions from each component. The red and orange curves represent the NEI models for the Fe and IME components, respectively. The magenta dashed curves indicate the Gaussian added to the model. The model curves for the power-law component are drawn in blue.

the two values is too small to be measured with the present data, considering the fact that the morphology of the stripe is also changing with time (figure 3). On the other hand, if the stripes are located inside the shell, or far downstream the blast waves, the transverse velocity of the stripes can be different from the above case. The proper motion of some structures perpendicular to the shock normal (figure 1b) would be in favor of this scenario. Models proposed in literature place the stripes in different locations (see e.g., Bykov et al. 2011; Malkov et al. 2012; Caprioli \& Spitkovsky 2013; Laming 2015). A future Chandra observation at another epoch would be needed to measure the proper motion of the stripes precisely enough to give meaningful comparison with that of the blast waves, which is a key to pinning down the line-of-sight locations of the stripes.

Our spectroscopy of each stripe at each epoch has revealed anti-correlation between surface brightness and photon indices (figure 5). In order to see whether or not the synchrotrondominant rim emission has a similar anti-correlation as well, we extracted spectra from regions R1-R5 defined in figure 1c. Since thermal emissions are negligible in these regions, we fitted the spectra with an absorbed power law and plotted the result in figure 6 together with those from the stripes as observed in 2009. In contrast to the stripes, the data points from the rim do not show a significant anti-correlation. Also, the rim emission is softer than the stripes with photon indices of $\Gamma=2.7-2.9$ as compared to $\Gamma=2.1-2.6$ of the stripes. This is consistent with the result by Lopez et al. (2015), who found the hardest $>10 \mathrm{keV}$ emission with NuSTAR in the west of the SNR coinciding with the location of the stripes.

Then what makes the spectra of stripes harder? Since the $\mathrm{X}$-ray band corresponds to the cutoff region of a synchrotron spectrum, photon indices reflect the cutoff energy $\left(\varepsilon_{0}\right)$ : a harder 


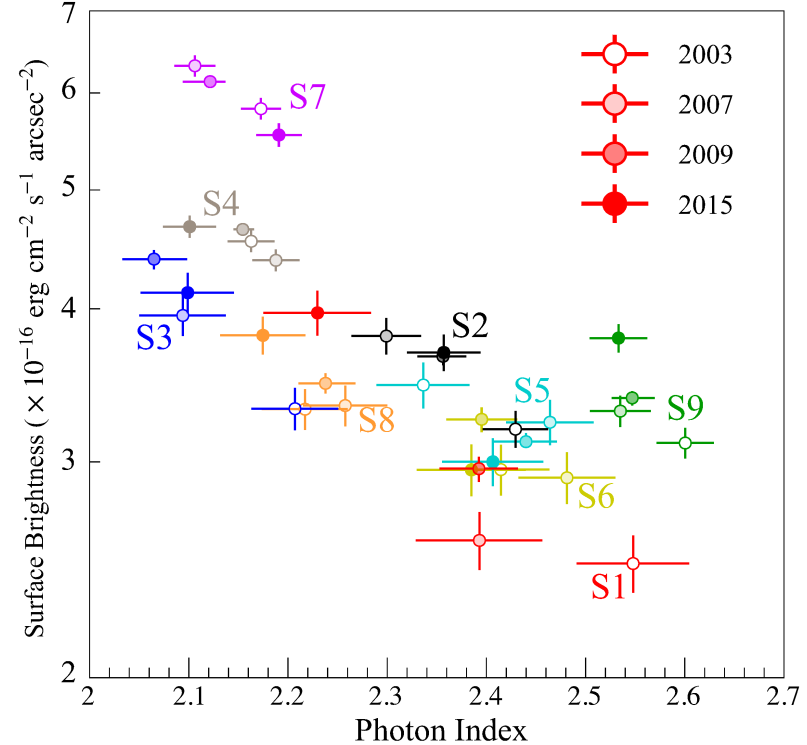

Fig. 5. Relation between the surface brightness and the photon indices in the stripes. The data points from each stripe are plotted in different colors. The color tint indicates the epochs of the data points.

spectrum means a higher $\varepsilon_{0}$ and vice versa. Let us first discuss the case in which stripes are associated with structures of the blast wave region although distinct spectra characteristics between the stripes and blast waves (figure 6) make this case less likely. According to the NuSTAR result by Lopez et al. (2015), the highest photon energy of synchrotron emission in Tycho's SNR seems to be limited by its age (= acceleration time). Given that, the cutoff energy depends both on the shock velocity and on the magnetic field strength as $\varepsilon_{0} \propto v_{\mathrm{sh}}{ }^{4} B^{3}$ (Lopez et al. 2015). Thus, the hard spectra of the stripes can be ascribed to fast shock velocity and/or strong magnetic field of the region. Considering the peculiar morphology of the stripes, it would be rather unlikely that only a fast shock velocity accounts for the hardness. Instead, it would be more probable that the magnetic field is amplified in the stripes through, for example, the resonant (Skilling 1975) or non-resonant (Bell 2004) cosmicray streaming instability. The problem about this scenario is that we cannot explain the short time variability of the stripes at the same time. The timescale of the variability expected in the age-limited case would be in the order of the age of the SNR, $\sim 100 \mathrm{yr}$, which is much longer than observed. One of the possible solutions that can reconcile with both the result by Lopez et al. (2015) and the variable stripe emissions would be that synchrotron emissions of most of the regions are age-limited whereas those right at the stripes are loss-limited with the amplified magnetic field.

If the stripes are not projections of blast waves but are located far downstream of the shock, the hard spectra are somewhat puzzling. After being accelerated at the blast wave, electrons are transported downstream through advection or diffu-

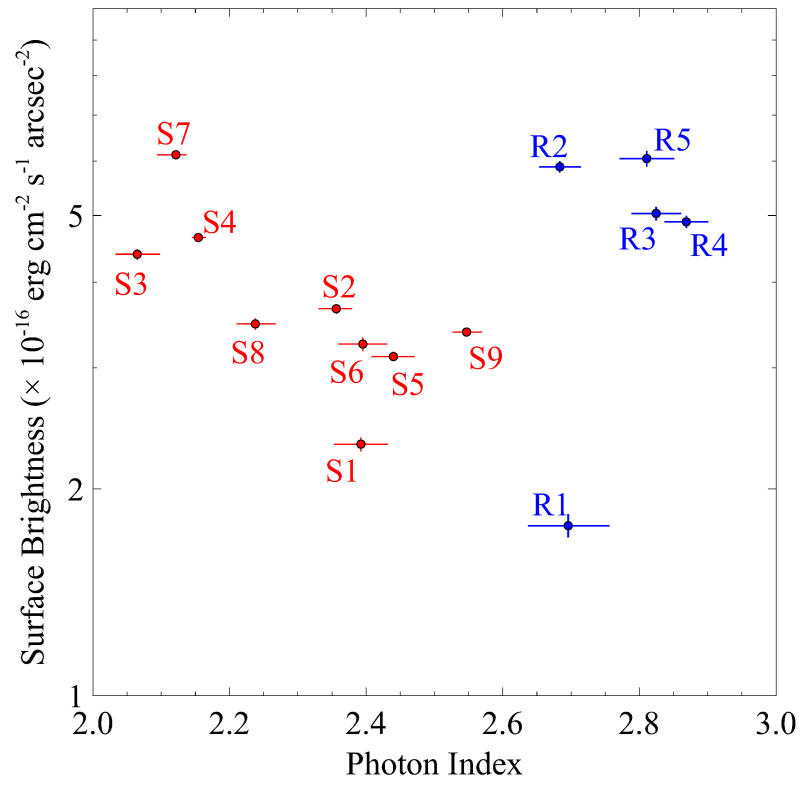

Fig. 6. Comparison of the relation between the stripes (red) and the rim (blue). The data points plotted here are from the observations in 2009.

sion. While being transported, ultra-relativistic electrons lose their energies via severe synchrotron cooling loss, which makes the electron spectrum and thus synchrotron X-ray spectrum softer. Such softening is indeed observed in Tycho's SNR by Cassam-Chenaï et al. (2007) with Chandra. One of the possible mechanisms to make the synchrotron spectra harder in the stripes is boosting synchrotron photon energy with a strong magnetic field. Since synchrotron photon energy $(\varepsilon)$ is related to parent electron energy $\left(E_{\mathrm{e}}\right)$ as $\varepsilon \propto B E_{\mathrm{e}}{ }^{2}$, a stronger magnetic field can make the synchrotron cutoff energy higher and thus synchrotron spectra observed with Chandra harder. In addition, if compressible waves/turbulence is present in the stripes, stochastic acceleration may occur and electron spectra would become even harder. We note that Zhang (2015) theoretically studied such a scenario.

In either case of the two discussed above, it would be very challenging to explain also the anti-correlation between surface brightness and photon indices (figure 5). The result would indicate only a small number of parameters are responsible for the temporal and spatial variation of the stripe emission otherwise such a tight anti-correlation would not appear. Another fact to note about the result in figure 5 is that the surface brightness is similar between stripes. This suggests that line-of-sight depths of the stripes are similar to each other, under an assumption that the nine stripes have similar magnetic field strengths and relativistic electron densities. If so, it would be more natural to consider that the stripes are shaped like spheroids rather than thin sheets. 


\section{Conclusions}

Using Chandra data obtained in 2003, 2007, 2009, and 2015, we searched for temporal and spatial variation of synchrotron radiation of the stripes in the southwestern region of Tycho's SNR in a more systematical way than the work by Okuno et al. (2020). Our imaging analysis revealed time variability of the emission in this region. Analyzing spectra of nine bright stripes, we found significant time variabilities not only of the stripe S1 previously reported by Okuno et al. (2020) but also of other stripes S2, S3, S7, and S9. If we attribute the flux increase to production of X-ray emitting electrons through diffusive shock acceleration and the flux decrease to synchrotron cooling of electrons, the observed time variabilities indicate that the magnetic field is amplified to $\sim 500 \mu \mathrm{G}$. The spectra of the stripes were found to be harder $(\Gamma=2.1-2.6)$ than those of the rim $(\Gamma=2.7-2.9)$, which would also be explained by amplified magnetic fields and/or stochastic acceleration in the stripes. Another finding is a tight anti-correlation between the surface brightness and photon indices of the stripe emission, which would indicate that only a small number of parameters control the temporal and spatial variation of the stripe emission.

\section{Acknowledgments}

This work is supported by JSPS/MEXT Scientific Research Grant Numbers JP25109004 (T.T. and T.G.T.), JP19H01936 (T.T.), JP26800102 (H.U.), JP19K03915(H.U.), and JP15H02090 (T.G.T.).

\section{References}

Ackermann, M., Ajello, M., Allafort, A., et al. 2013, Science, 339, 807

Aharonian, F., Akhperjanian, A. G., Bazer-Bachi, A. R., et al. 2007, A\&A, 464, 235

Arias, M., Vink, J., Zhou, P., et al. 2019, AJ, 158, 253

Archambault, S., Archer, A., Benbow, W., et al. 2017, ApJ, 836, 23

Arnaud, K. A. 1996, Astronomical Data Analysis Software and Systems $\mathrm{V}, 17$

Bamba, A., Yamazaki, R., Ueno, M., et al. 2003, ApJ, 589, 827

Bell, A. R., \& Lucek, S. G. 2001, MNRAS, 321, 433

Bell, A. R. 2004, MNRAS, 353, 550

Blandford, R., \& Eichler, D. 1987, Phys. Rep., 154, 1

Borkowski, K. J., Reynolds, S. P., Williams, B. J., et al. 2018, ApJL, 868 , L21

Bykov, A. M., Ellison, D. C., Osipov, S. M., et al. 2011, ApJL, 735, L40

Caprioli, D., \& Spitkovsky, A. 2013, ApJL, 765, L20

Cassam-Chenaï, G., Hughes, J. P., Ballet, J., et al. 2007, ApJ, 665, 315

Eriksen, K. A., Hughes, J. P., Badenes, C., et al. 2011, ApJL, 728, L28

Foster, A. R., Smith, R. K., \& Brickhouse, N. S. 2017, Atomic Processes in Plasmas (apip 2016), 190005

Garmire, G. P., Ricker, G. R., Bautz, M. W., et al. 1992, AIAA/AAS Astrodynamics Conference

Giuliani, A., Cardillo, M., Tavani, M., et al. 2011, ApJL, 742, L30

Hwang, U., Decourchelle, A., Holt, S. S., et al. 2002, ApJ, 581, 1101

Jogler, T., \& Funk, S. 2016, ApJ, 816, 100
Koyama, K., Petre, R., Gotthelf, E. V., et al. 1995, Nature, 378, 255

Lagage, P. O., \& Cesarsky, C. J. 1983, A\&A, 125, 249

Laming, J. M. 2015, ApJ, 805, 102

Lopez, L. A., Grefenstette, B. W., Reynolds, S. P., et al. 2015, ApJ, 814, 132

Malkov, M. A., \& Drury, L. O. 2001, Reports on Progress in Physics, 64, 429

Malkov, M. A., Sagdeev, R. Z., \& Diamond, P. H. 2012, ApJL, 748, L32

Okon, H., Tanaka, T., Uchida, H., et al. 2020, ApJ, 890, 62

Okuno, T., Tanaka, T., Uchida, H., et al. 2020, ApJ, 894, 50

Sato, T., \& Hughes, J. P. 2017, ApJ, 840, 112

Skilling, J. 1975, MNRAS, 172, 557

Tanaka, T., Uchiyama, Y., Aharonian, F. A., et al. 2008, ApJ, 685, 988

Uchiyama, Y., Aharonian, F. A., Tanaka, T., et al. 2007, Nature, 449, 576

Uchiyama, Y., \& Aharonian, F. A. 2008, ApJL, 677, L105

Warren, J. S., Hughes, J. P., Badenes, C., et al. 2005, ApJ, 634, 376

Williams, B. J., Chomiuk, L., Hewitt, J. W., et al. 2016, ApJL, 823, L32

Wilms, J., Allen, A., \& McCray, R. 2000, ApJ, 542, 914

Yamaguchi, H., Hughes, J. P., Badenes, C., et al. 2017, ApJ, 834, 124

Zhang, M. 2015, ApJ, 812, 148

Zhou, P., Chen, Y., Zhang, Z.-Y., et al. 2016, ApJ, 826, 34 